\title{
Aiding Disabled Patients using Motion Based Message Conveyor
}

\author{
J Caroline El Fiorenza, Barbie Das, Kirthika Sivakumar, M Reene Stephanie
}

\begin{abstract}
This is an innovative application that can help the disabled or paralytic patients to convey messages or call helper or the nurse just by using simple gesture movements since paralytic or disabled people cannot press a button or ring to call a nurse or helper when they need. Even if they can move a particular limb like moving their hand up to a limit or moving their legs up to a limit, this system can be mounted on those limbs and just by a simple movement or a gesture, and they can pass on various messages and call the attendant. The reason why this prototype is needed is described by the following objective. The primary objective of this project is implementing a relatively less expensive reliable framework that helps in assisting the establishment of communication between immobile or disabled people and their caretaker or nurse. The method how to do is described here. Messages can be easily sent to the nurse or caretaker simply by tilting the accelerometer that is joined with the mobile body part. The tilt angle is then sent to a controller which then communicates between the disabled person and the caretaker. It also takes appropriate decision to send the respective message according to the corresponding tilt. The message that is to be delivered can be shown on the LCD display. When the message is being displayed, the buzzer goes off to alert the caretaker or concerned person in case of any emergency. Hence project gives an effective, easy and a vital solution for many kinds of issues that the caretakers and nurses face for communicating with the patients that are disabled or immobile.
\end{abstract}

Keywords: Paralytic, patient-nurse, communication, ATMega microcontroller

\section{INTRODUCTION}

In the medical sector, among the enormous number of improvements done, only a few of them actually are concerned with the helping of the disabled patients for communication. Despite the fact that monitoring systems make it less complex for doctors to gather and supervise a patient's vitals, there aren't many alternative solutions for the disabled patients to communicate in an actual verbal manner.

Revised Manuscript Received on April 25, 2020.

* Correspondence Author

J Caroline El Fiorenza*, Assistant Professor, Department of Computer Science and Engineering, SRM Institute of Science and Technology, Chennai, India.

Barbie Das, B Tech, Department of Computer Science and Engineering, SRM Institute of Science and Technology, Chennai, India.

Kirthika Sivakumar, B Tech, Department of Computer Science and Engineering, SRM Institute of Science and Technology, Chennai, India

M Reene Stephanie, B Tech, Department of Computer Science and Engineering, SRM Institute of Science and Technology, Chennai, India

(c) The Authors. Published by Blue Eyes Intelligence Engineering and Sciences Publication (BEIESP). This is an open access article under the CC BY-NC-ND license (http://creativecommons.org/licenses/by-nc-nd/4.0/) trustable way rather than using the patient-nurse communication with a conventional approach. In the present situation, the patient must be subject to a relative or generally To solve this particular problem, an easier yet effective method is proposed here. The major objective here is to implement a modern technique using technologies providing a quicker and a caretaker both of which need to go to the patient continually. The current goal is making the disabled patients free to be able to communicate with their nurse by the simple action of tilting the device which is situated on their hand or some other part of the body which is mobile.

For this purpose, there is a transmitting media and a receiver unit. The receiver unit may be mounted on the nurse's collars or near the doctor whoever is in charge of handling the patient. The transmitter unit consists of an accelerometer. The accelerometer is used to track the gesture movements which are connected to an ATMega family microcontroller. The microcontroller then transmits this message through the RF transmitter to the receiver unit. The receiver unit consists of an 8051 family microcontroller which then decodes the received messages and displays it on the LCD screen also sounds a buzzer to indicate that the patient is in need of help and displays which kind of help the patient requires depending on the gesture movement of the patient.

This is not only easy for the patient, but also stress-free for the caretakers at the same time. After the message being sent to the caretaker, the monitoring of their requests become very easy and thus attending to them is also done without any further delay. In case of any emergency, the buzzer alerts the caretakers. To create a more dynamic system, various ideas were pooled together to create a smart system in the view of making patients a little more self-dependent also making assistance simpler and easier for caretakers, nurses or family members.

\section{LITERATURE SURVEY}

A mechanism to detect hand gestures in midair by using a real-tome hand gesture recognition system which is capable of controlling the device in accordance to the input gesture is presented here. This primary thought is to adapt a methodology for the physically disabled patients to control the gadgets or appliances using their hand gestures without the need to go to the main switchboard. In this system, the hand gestures in the form of sign languages are taken as input and converted to characters or symbols by the computer when the hand gestures are being made in mid-air. The technique used in this system is image processing to record the hand gestures of the patients.

The advantage of this system is that it is quite

Published By:

Blue Eyes Intelligence Engineering DOI: 10.35940/ijeat.D8381.049420

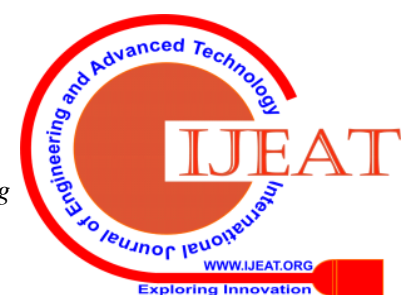


convenient for the patients to do activities independently without any sort of assistance

whereas on the other side, this system might display error in the detection of the perfect gesture due to change in factors like brightness which depicts the inaccuracy of the system. [1]

A smart attender calling system for the purpose of efficient monitoring of patients who are not capable of verbal communication is presented here. Here, a device was created wherein a flex sensor is mounted on the glove platform corresponding to the position of the fore-finger. Upon the movement of the finger of the patient, the flexion induces a variation in the voltage. When the comparative voltage ratio goes above a certain magnitude, the signal is passed onto the APR9600 which plays the pre-recorded voice message. The technology used here is the aforesaid system along with the flex sensor. This system does not require any sort of complicated signal processing because the bend angle of the sensor is proportional to the output signal of the displacement sensing module. At the same time, this system is quite fragile and the soldering on the flex sensor is certainly a difficult task to be performed in this system. [2]

An emergency embedded framework based on SIP called as SEEK is presented here. This enables the devices that are embedded to trigger the call to emergency regions as well as support real time communication based on IP. The SEEK framework contains a haptic device along with a few body sensors that enables the disabled and old people to make emergency calls who live independently. In this prototype, the sensors that are embedded in the SEEK aggregate the vital signs that monitor the patients' present health condition which triggers the emergency call thus providing sensor data that is real-time and continuous advantage of this prototype is that it creates a special type of communication which focuses on the touch and thus takes advantage of the user's sense of touch and feeling. Though this is an advanced technology, the implementation of this technology is quite expensive which makes it unaffordable for most patients. [3]

A post-stroke rehabilitation system which is based on applying the tilt sensors for the hemi paretic-arm patients is presented here. It contains all the development, simulation and the design of the results. The result is then recorded for the reason of progressive tracking of status and post-processing purpose. Here, the patient wears a set of sensors on the hand or palm when doing some movements with the arm. This data is then converted into series of readable data which is then displayed on the screen of the corresponding smartphone. The proposed system uses the Arduino Uno Tilt Sensor along with Bluetooth. It is a merit that this system provides more information than many other conventional methods. This system improves the quality, results and patient's progress as well. The only disadvantage would be that since the Arduino uses $\mathrm{C} / \mathrm{C}++$ with a few small extensions of its own, lots of libraries need to address issues like Ethernet and ZigBee. [4]

A hand-held device which is capable of computing the stiffness while having interaction with any soft object is presented here. This multi-indenting system measures the stiffness of the object and also the tilt and pan angles between the surface of soft object and the sensor. Palpation is one of the medical diagnosis techniques the size, location, texture and the consistency of the various areas of tissue are examined by doctors by combining vision with the sensation received from their fingertips when coming in contact with a soft tissue. This information is hence utilized for the purpose of diagnosis. This system uses four linear indenters and a USB camera for implementation. This system is highly efficient, contains low thermal dissipation and can handle large output current. On the other side, it is a complicated design with a high cost factor. [5]

A prototype on a glove based motion capturing system using fingers is presented here. In this system, a high speed capturing is achieved which is sufficient to represent the motions of fingers irrespective of smooth or swift quite economically. This system works for approximately 14 channels at a speed of $54 \mathrm{~Hz}$ with flexible strain sensors and signal conditioning circuits. For the detection of motion, stretchable strain sensors that are highly linear are utilized. The merit of this system is that this system has a one-touch calibration mode for baseline cancellation making this whole system more user friendly. In addition to this, this system has accuracy, flexibility, isolation and a built-in front-end. The only drawback of this system is that it is quite expensive and has high chances of committing a high rate of errors. [6]

\section{MODULE DESCRIPTION}

Accelerometer is associated with Arduino Uno controller that gives input or information. The accelerometer is the core of this system. This can be the two pivots or the three hub static accelerometer associated with analog input of controller. To detect the speed which is increasing, it is interfaced or associated with the controller. The second phase of the framework is the controller. The data is processed by the controller which then determines the appropriate message according the conditions being satisfied. The message is then further sent to the LCD. The LCD displays the corresponding instruction. The instruction will then be given to the speakers or speech module at the same time. This helps the patient have knowledge by making them hear on whether the appropriate instruction or command has been sent or not. Sometimes, the messages that are necessary for the patients to deliver may be stored in the SD Card/ memory card. Simultaneously, the nurses, doctors or relatives of the patient may be alerted when the buzzer goes on.

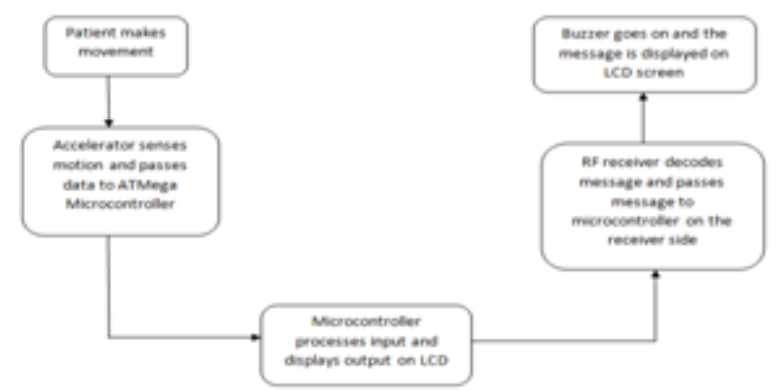

Fig. 1 Architecture diagram

\section{A. Transmitter}

The transmitter module consists of all the necessary parts required to transmit signals.

The transmitter is mounted on the hand of the disabled or paralytic person. The 
transmitter contains an accelerometer that measures the statistics of motion. The patient motion recorder device consists of an RF transmitter in order to transfer the data signal. Data signal is then sent to the micro controller.

\section{B. Processor}

The processor module consists of the ATMega microprocessor that processes the data of signal. The micro controller processes the data. It displays the particular message as per input obtained. On the LCD screen, the associated message is then displayed by the micro controller. It also sounds a buzzer along with message as soon as it receives motion signal from the accelerometer.

\section{Receiver}

The receiver module consists of all the parts that receive the transmitted signals that are processed by the ATMega microprocessor. On the other side, the RF receiver receives the data from the transmitter side's micro controller. The receiver then decodes the input data received, before passing it to the micro controller. The micro controller on the receiver side processes the input and responds to it.

Hardware Specifications:

- 8051 and ATMega Microcontroller for processing

- Transformer/Adapter provides power

- IC and its IC sockets

- Capacitors to store energy

- PCB and Breadboards

- Switch, Cables and Connectors

- Diodes and LED for the light

- Transistors for transmission

- Accelerometer for detecting angle

- Push Buttons

- Resistors and diodes

Software Specifications:

- MC Programming Language: C

- Keil $\mu$ Vision IDE

- Avr Studio

\section{IMPLEMENTATION}

\section{A. 8051 Microcontroller}

In 1981, Intel had designed the 8051 microcontroller. This is an 8-bit microcontroller. There are 40 DIP (Dual Inline Package) pins, a storage capacity of $4 \mathrm{~kb}$ of ROM and 128 bytes of RAM storage and two 16-bit sized timers. As per the requirement, the 8051 microcontroller consists of four parallel 8-bit ports that are programmable and addressable as well. In the microcontroller, an on-chip crystal oscillator having crystal frequency of $12 \mathrm{MHz}$ is integrated.

The 8051 Microcontroller consists of two buses in which one of the buses is for program and other for data. Since there are two buses, it has two storage rooms of $64 \mathrm{~K}$ by 8 sizes for both program and data. The 8051 microcontroller consists of an 8 bit processing unit and an 8 bit accumulator. It also contains an 8 bit $\mathrm{B}$ register as majorly functioning blocks. Also, the Keil software is used for the programming part with embedded $C$ language. This microcontroller also consists of several other 8 bit and 16 bit registers.

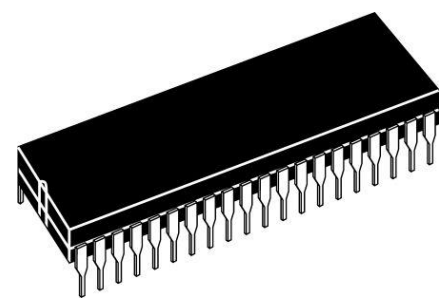

Fig. 28051 Microcontroller

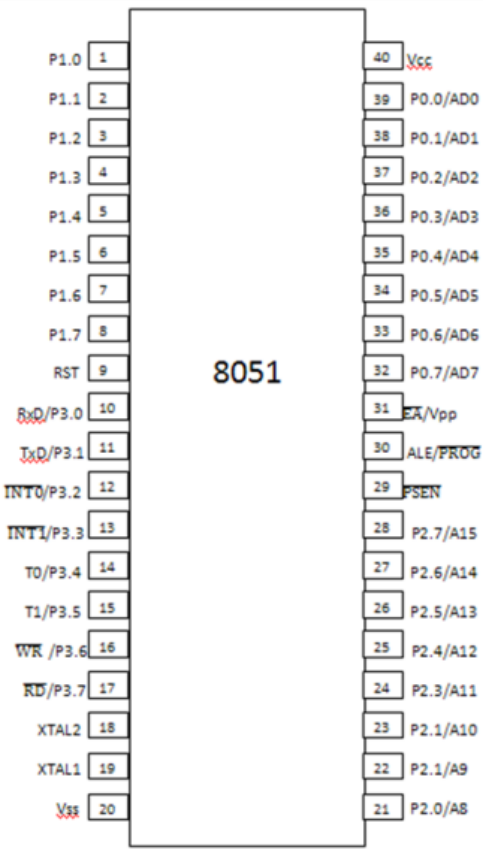

Fig. 3 Architecture Diagram of 8051 Microcontroller

\section{B. ATMega Microcontroller}

The ATMega Microcontrollers were manufactured by the Atmel Corporation. With the Reduced Instruction Set (RISC) based on the Harvard Architecture, the ATMega microcontroller is of 8-bit. The standard features of ATMega microcontroller includes Timers, data EEPROM (Electrical Erasable Programmable Read Only Memory), on-chip ROM (Read Only Memory), Ports for Input and Output , Data RAM (Random Access Memory) which also consists of extra peripherals such as Serial Interface Ports, Analog to Digital Converters (ADC) and many other ports. There are approximately 120 or more program memory and instruction set ranging from $4 \mathrm{~K}$ to $256 \mathrm{~K}$ Bytes. Since the ATMega microcontrollers are Harvard Architecture based, they are separate program memory and data memory. The Program memory which is also known by other names such as Code Memory or Program is nothing but the Flash Random Access Memory (ROM).

The Program Memory is having a size ranging from $8 \mathrm{~K}$ to $128 \mathrm{~K}$ Bytes. The data memory can be widely classified into three segments - Input/output memory, Internal Static Random Access Memory (SRAM) and 32 General Purpose Registers.

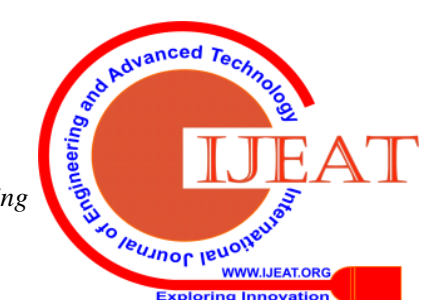



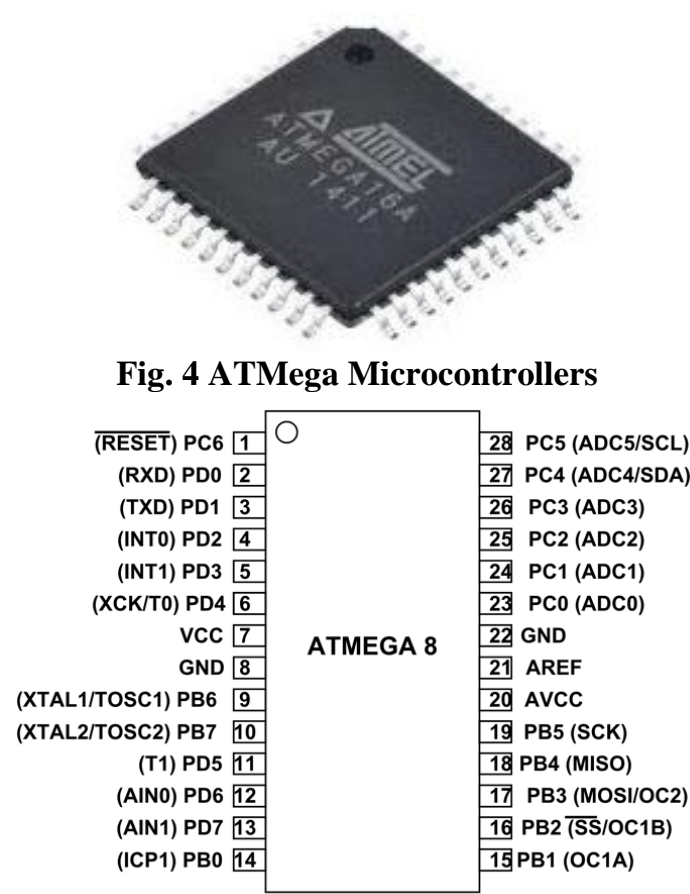

Fig. 5 Architecture Diagram of ATMega Microcontroller

\section{Keil $\mu$ Vision IDE}

In a single environment, the $\mu$ Vision IDE is capable of combining facility building, editing of source code, run-time environment, project management and debug process of a program. This particular IDE is really easy-to-use and it helps in accelerating the development of embedded software. In the Keil $\mu$ Vision IDE, the user is allowed to create individual window layouts anywhere on the visual surface and this IDE supports multiple screens making it even more convenient for the users. There is single environment which is provided by the $\mu$ Vision Debugger that allows users in testing, verifying and optimizing the application code. The authentic features of debugger includes having breakpoints both simple and complex, watch windows, providing control for execution and when it comes to device peripherals, it provides full visibility. By using the device support from Software Packs and software components that are pre-build, an application can be created on the Run-Time Environment in the $\mu$ Vision Project Manager. These software components include templates for source codes, documentation, and files for configuration, source modules and various libraries. For supporting wide ranges of devices and applications, the software components are mostly generic in nature.

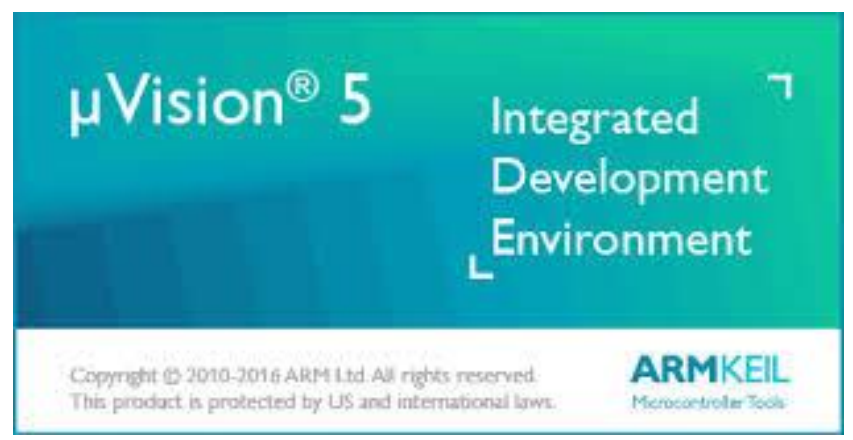

Fig. 6 Keil $\mu$ Vision IDE
Fig. 4 ATMega Microcontrollers

\section{Avr Studio}

For the sole purpose of the development and debugging process for all the applications of AVR and SAM microcontroller, this integrated development platform (IDP) called Studio 7 was created. The Atmel Studio 7 IDP provides its users to perform tasks or operations such as writing, building and debugging applications that are written in $\mathrm{C} / \mathrm{C}++$ or any sort of code in assembly language in an easy-to-use environment which is seamless. For those programmers, debuggers and development kits supporting the AVR and SAM devices the connection is given seamlessly in the IDP.

The various features of the Avr studio are as follows:

- This studio supports more than 500 devices of AVR and SAM

- AVR Studio provides a huge source of code libraries that includes communication stacks, drivers, more than 1600 examples of projects with the source code.

- With the Advanced Software Framework (ASF), graphic service and functionalities of touch are provided.

- Through the Gallery of Atmel various IDE extensions, for developing tools and embedding software from Microchip.

- Atmel QTouch Composer aids in: validating performance of system, power consumption of monitor, tuning touch designs and graph tracing.

- When the Wireless Composer runs on target, the test performance of the wireless design can be configured.

- The assembly code and the $\mathrm{C} / \mathrm{C}++$ codes, with the help of integrated compiler can be written and debugged

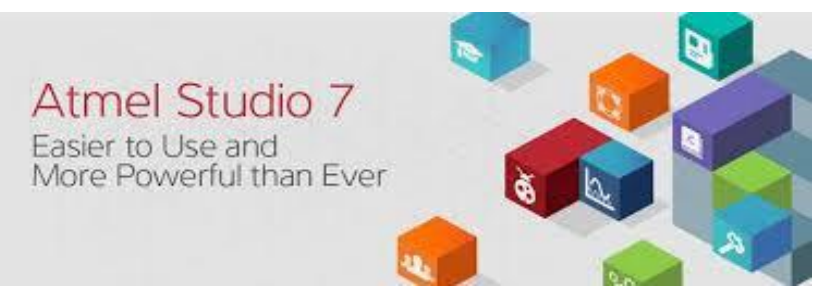

Fig. 7 Avr studio

\section{E. Accelerometer \\ a) Definition:}

A device that can calculate acceleration properly is called as accelerometer. Proper coordinate acceleration and a body's acceleration (or the rate of change of velocity) is not the same. For instance, acceleration due to Earth's gravity can be calculated by placing an accelerometer at rest on the Earth's surface, straight up (by definition) of $g \approx 9.81 \mathrm{~m} / \mathrm{s} 2$. By comparison, a free fall accelerometer (falling at a rate of about $9.81 \mathrm{~m} / \mathrm{s} 2$ towards the center of the Earth) would test null.

\section{b) Features:}

Accelerometers provide features such as free fall detection that can be used in the Active

Hard Drive Protection, tap detection that is practical for low power applications, temperature adjustment and $0-\mathrm{g}$ range sensing.

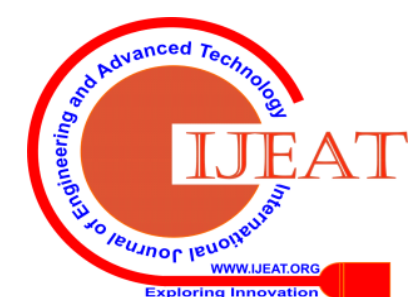




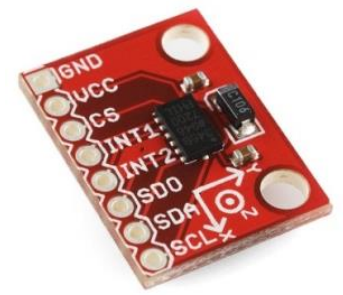

Fig. 8 Accelerometer

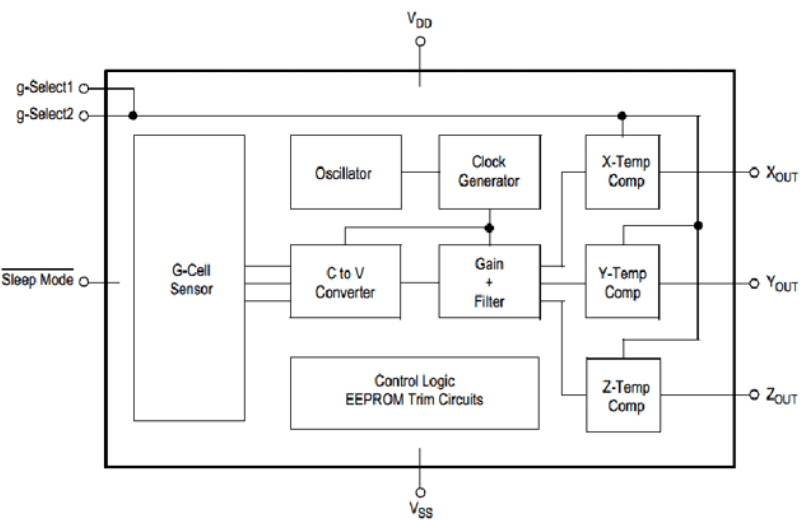

Fig. 9 Architecture Diagram of Accelerometer

F. Resistor

\section{a) Definition:}

A resistor has always been a passive electrical two-terminal component which implements electrical resistance as an element of a circuit. Resistors can minimize current flow in electronic circuits; divide voltages in circuits, change signal rates, bias active elements and terminate transmission lines, etc. For power distribution systems or a generator to test loads a high power resistor that can give several watts of electrical power is used. Fixed resistors include resistances which only slightly change with temperature, time or voltage of operation. Circuit elements (such as lamp dimmer or volume) can be modified with variable resistors or as sensing devices for humidity, power, chemical activity, heat or light.

\section{b) Features:}

Features of resistors are: It has a low coefficient of resistance, frequency dependency, noise and power rating. It insulates the wires and protects from influences from the environment. It can hold maximum voltage that is allowed and can be stable for a long term. It has good pulse stability, mechanical strength, tolerance towards resistance value and thermos electric effect.
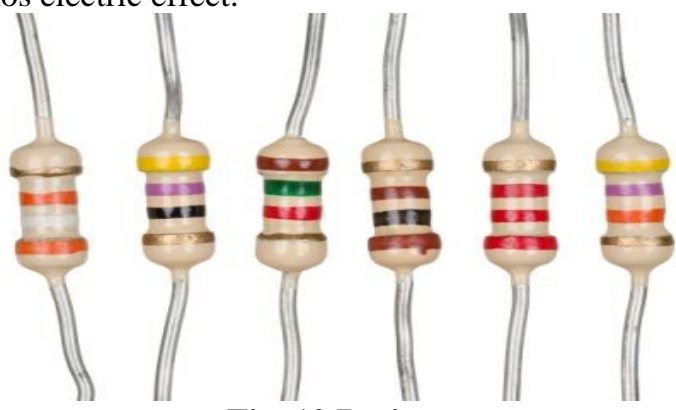

Fig. 10 Resistor

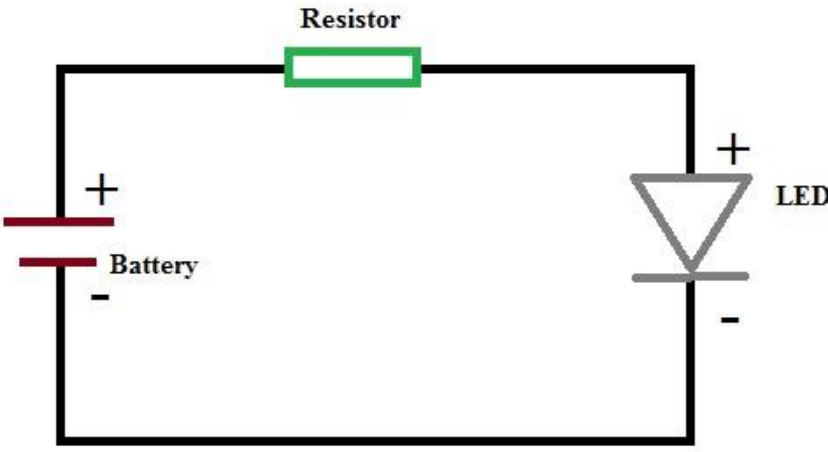

Fig. 11 Architecture Diagram of Resistor

G. Capacitor

a) Definition:

A capacitor is an apparatus that can store electrical energy in an electrical field. This is a passive electronic component with two terminals. The effect of a capacitor is called as capacitance. While some capacitance exists in proximity in a circuit between any two electrical conductors, capacitance can be added to a circuit by using a capacitor. Initially, the capacitor was a condenser or compensator. Still widely used in many languages, this name and its cognates, but seldom in English, with one exception that is condenser microphones which is also known as capacitor microphones.

b) Features:

Its features are Nominal Capacitance, Temperature Coefficient, Working Voltage, Leakage Current, Working Temperature, Tolerance, Polarization and Equivalent Series Resistance.

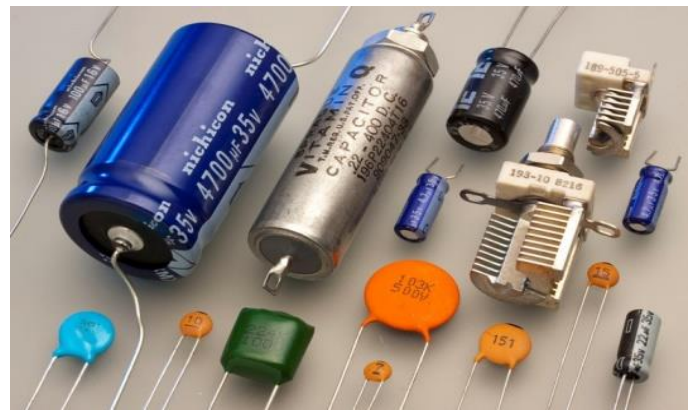

Fig. 12 Capacitor

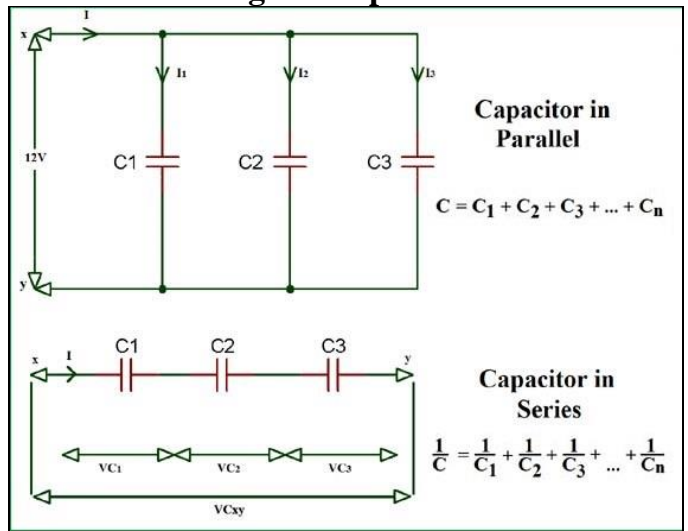

Fig. 13 Architecture Diagram of Capacitor

\section{H. Transistor}

a) Definition:

A device that is used to amplify or alternate electrical and electronic signals is called as a transistor. It is a

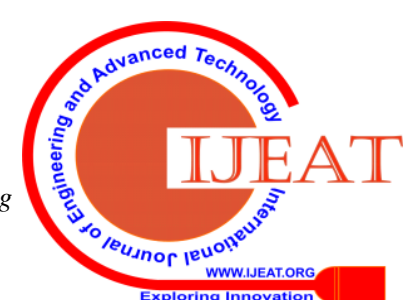


semiconductor material and has at least three terminals to connect to an external circuit. A voltage or current applied to one pair of terminals on the transistor regulates the current via another pair of terminals. Since the controlled (output) power can surpass the controlling (input) power, a transistor may amplify a signal. Most of the transistors are usually found in integrated circuits but some of them can be individually packed.

\section{b) Features:}

Features of transistor are high voltage, high current, good switching characteristics, and high driving power.

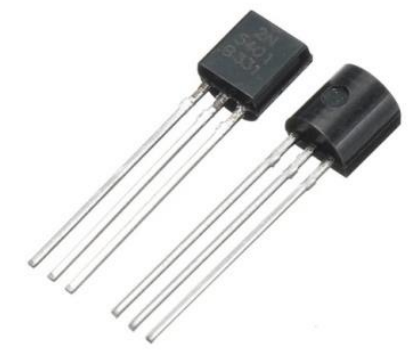

Fig. 14 Transistor

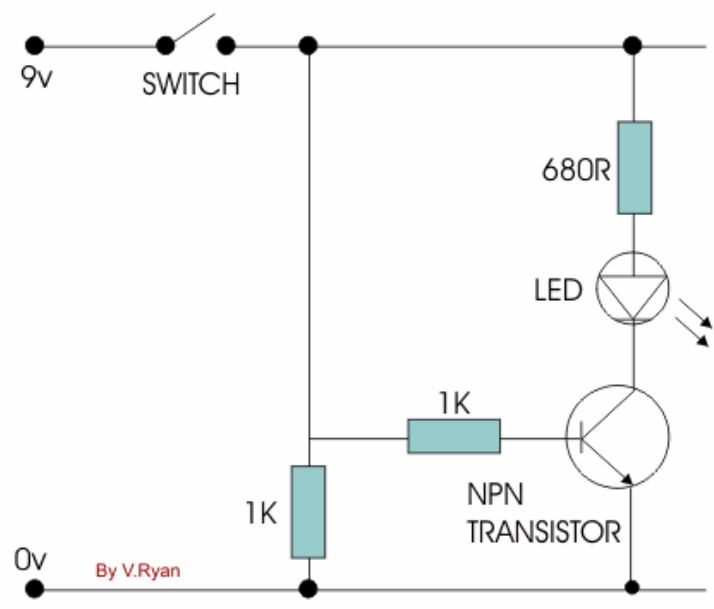

Fig. 15 Architecture Diagram of Transistor

\section{Diodes}

\section{a) Definition:}

A device that can mainly conduct current in one direction: has low resistance in one direction and high resistance in the other direction is called as a diode. A diode vacuum tube or thermionic diode is a two-electrode vacuum tube, a heated cathode and a plate in which electrons can migrate from cathode to plate in one direction only. A semiconductor diode is made up of a crystalline piece of semiconductor material that has a $\mathrm{p}-\mathrm{n}$ junction connected to two electrical terminals, the most widely used form today. The first electronic device with semi-conductors is the semiconductor diodes. In 1874 German physicist Ferdinand Braun made the discovery of asymmetric electrical conduction across the interaction between a crystalline mineral and a surface. Some diodes today are made of silicon, but it can also be made with gallium arsenide, germanium and other materials.

\section{b) Features:}

Depending on the voltage applied over it a diode will operate in one of three regions:

1. Forward bias: If the voltage is positive across the diode, the diode is "on" and the current will pass through it. The voltage should be higher than the forward voltage (VF) so that the current is anything relevant.

2. Reverse bias: This is the diode's "off" mode, where the voltage is smaller than VF but greater than -VBR. Current flow in this mode is (mostly) blocked, and the diode is off. A very small number of current (on the order of nA) - called the current of reverse saturation - is able to flow through the diode in reverse.

3. Breakdown: When the voltage applied across the diode is very high and negative, lots of current will flow backwards (in the reversed direction), from cathode to anode.

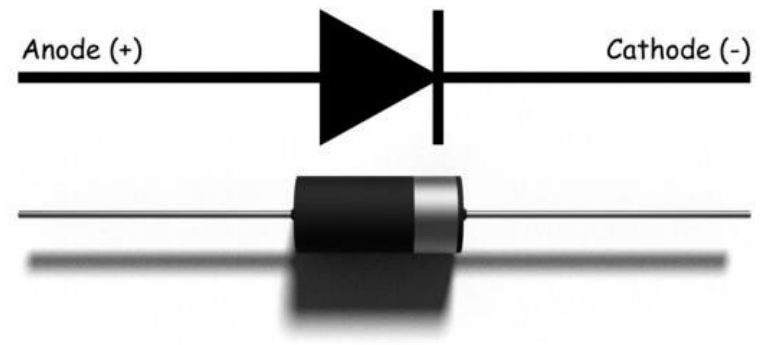

Fig. 16 Diode

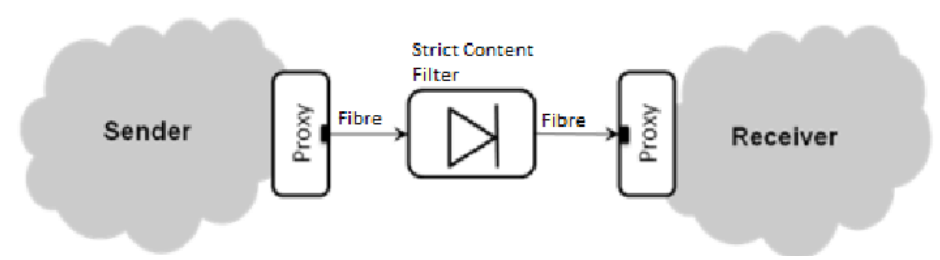

Fig. 17 Architecture Diagram of Diode

\section{J. Switch}

\section{a) Definition:}

A switch is an electrical part capable of disconnecting or linking the conduction path in an electrical circuit, interrupting or diverting the electrical current from one conductor to another.

The most common type of switch is an electromechanical switch that has one or more sets of mobile electrical contacts connected to external circuits. Current will pass only when a pair of contacts touches and no current will flow when the contacts are separated.

\section{b) Features:}

Key features include switching function, voltage, and current ratings, as well as various e-stop applications-specific features such as broken cable detection. For manual control of the overhead lights and fans a single pull switch can be used.

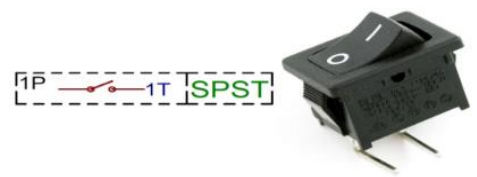

Fig. 18 Switch

Blue Eyes Intelligence Engineering

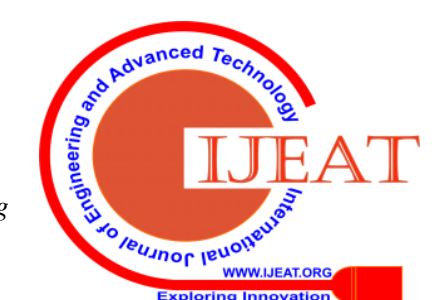




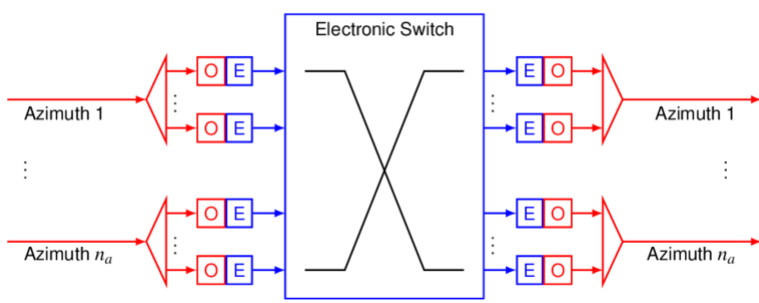

Fig. 19 Architecture Diagram of Switch

\section{K. Cables and Connectors}

\section{a) Definition:}

Cable: - A cable is made up of plastic and it is used for transmitting data between various devices or locations. A cable consists of two different types -a data cable and a power cable.

Connectors:-A connector is used to quickly connect or disconnect from a circuit path.

\section{b) Features:}

The lodging is the structure used to contain the terminals, ensure solidness of the associations and shield the electrical segments from short circuiting and ecological dangers. Physical measurements are breadths of the wire conduits, coats, temperature Rating, Curve Sweep, Flex Span, and Pulling Pressure. The connectors have two fundamental parts the lodging, and terminals for making the associations.

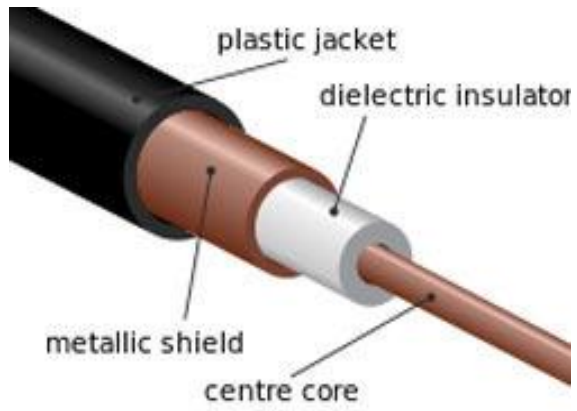

Fig. 20 Cable

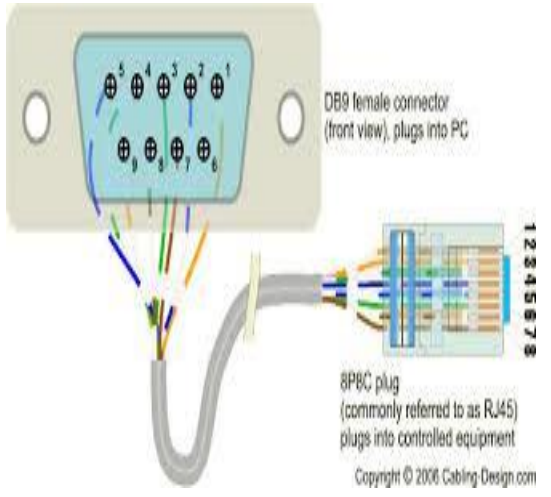

Fig. 21 Connector

\section{PCB and Breadboards}

\section{a) Definition:}

PCB- PCB is likewise called a Circuit Board. A PCB is a feeble board consists of fiberglass, composite epoxy, or other spread material. Pathways are engraved onto the board, particularly electrical portions on the PCB, for instance, transistors, resistors, and facilitated circuits.

Breadboards- Electronic circuit models are made up of Breadboards, the sheets can be reused for future purposes. The Breadboards are used to hold electronic components such as transistors, resistors, and chips together.

\section{b) Features:}

It helps when another person needs to comprehend and examine the circuit. Hues coding the jumper wires can help diminish disarray when fabricating a circuit. Adaptable PCBs helps in diminishing the board size, which makes it perfect for different applications where high sign thickness is required. It is essential to breadboard a circuit ought to be flawlessly and efficiently introduced; with the goal that one can troubleshoot it and make it run effectively and rapidly. PCBs help spare a great deal of room, likewise lessening the general board weight.

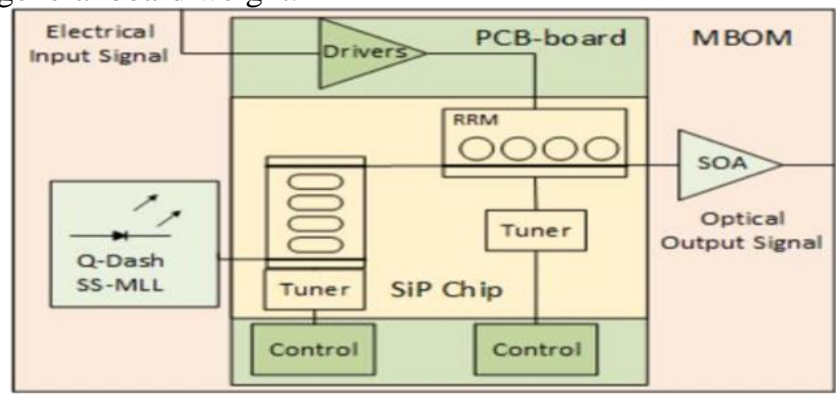

Fig. 22 Architecture Diagram of PCB

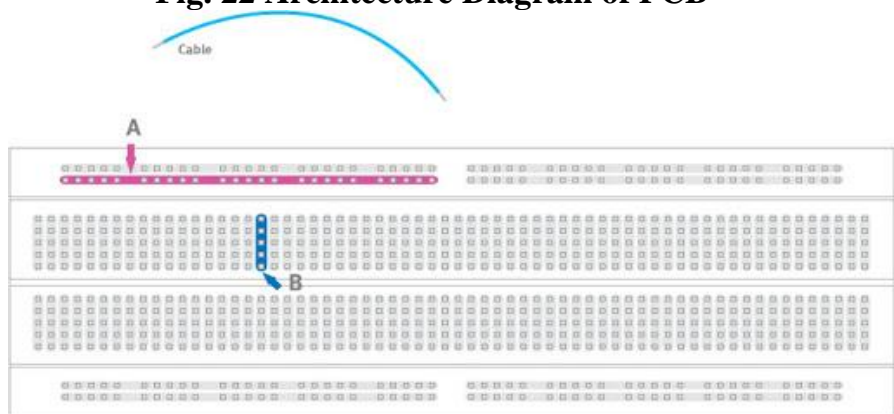

Fig. 23 Breadboard

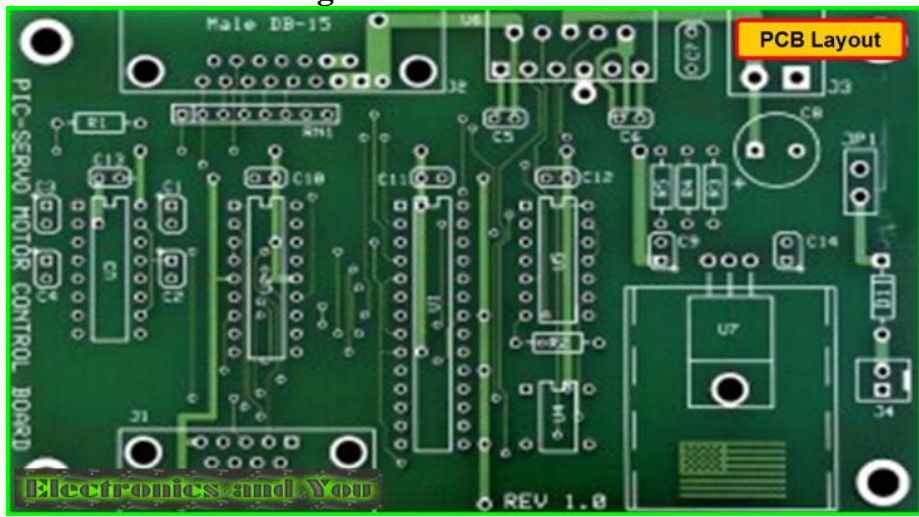

Fig. 24 PCB

\section{LED}

\section{a) Definition:}

LED is a $\mathrm{p}-\mathrm{n}$ junction diode that radiates light when connected. Marker lights are made up of LEDs. One of the major properties of LEDs is it consists of a two-lead semiconductor light source. LED has a major application in the growing industry of electronic components.

b) Features:

In 2005 the white LEDs arrived at yields of more than 30 lumens/Watt and hued adaptations 50 lumens/Watt. The light picks up keep on becoming expanding about each two years.

Long operational life as long as 50,000 hours. They don't emanate heat toward the lit up object they can be utilized to light up materials that blur

Published By:

Blue Eyes Intelligence Engineering

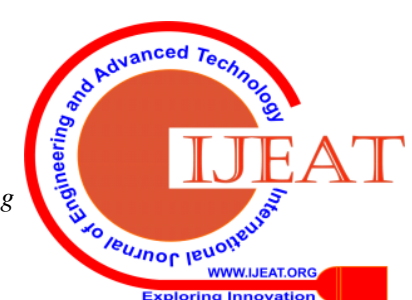


effectively like nourishment, masterpieces and so on. The LEDs are amazingly productive low vitality light sources. The LEDs don't produce bright (UV) or infrared (IR) radiation. Reduced light source, no other light has such little measurements for a similar light yield.

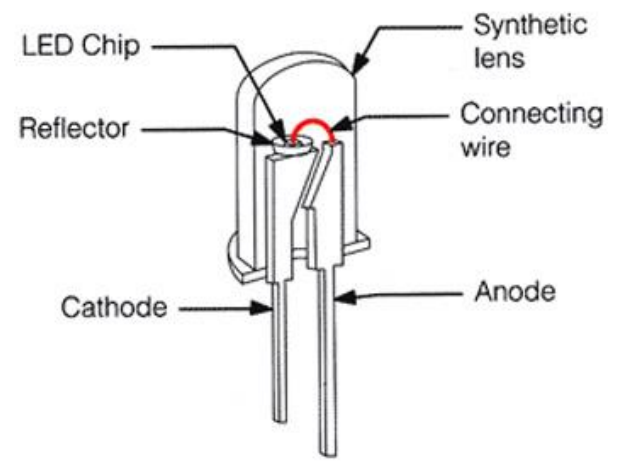

Fig. 25 LED

\section{N. Transformer}

\section{a) Definition:}

Electrical current can be transferred between the circles, without the actual contact between the two circuits. There are two types of transformer Step up transformer and step down transformer. The electrical energy moves from one circuit to another through the process of electromagnetic induction. An alternating current in any one coil of the transformer makes a movement which in turn activates an electromotive force control over some different coil of another transformer.

\section{b) Features:}

An alternating current in the winding generates movements inside the transformers. If the voltage is raised, the current is generally cut down and vice-versa. A transformer is an electronic component that uses Faraday's law and the ferromagnetic properties of iron to raise or lower cooling voltages.

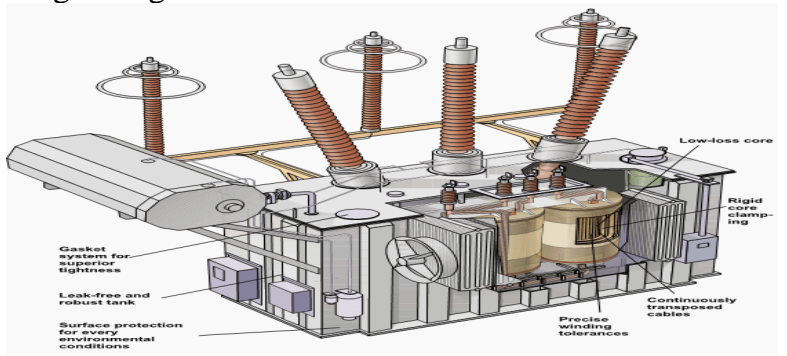

Fig. 26 Transformer

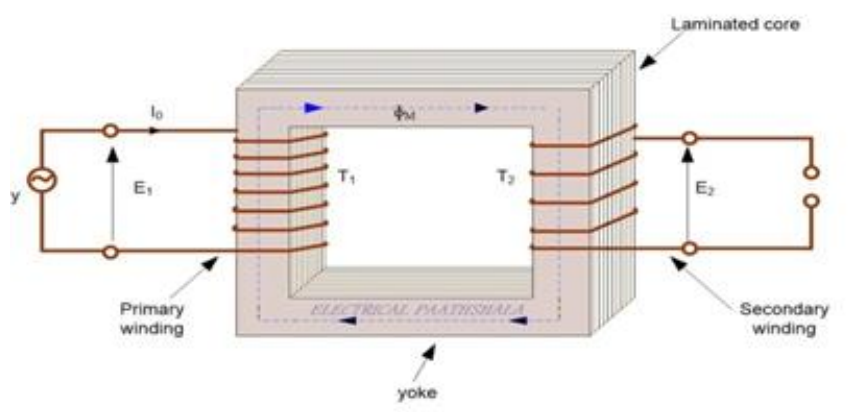

Fig. 27 Architecture Diagram of Transformer

\section{O. Push Buttons}

\section{a) Definition:}

In modern and business applications, press catches can be associated together by a mechanical linkage with the goal that the demonstration of pressing one catch makes the other catch be discharged. Press catches permit us to control the circuit or make a specific association just when we press the catch. A press button is additionally utilized for activating of the SCR by door terminal. Basically, it makes the circuit associated when squeezed and breaks when discharged.

\section{b) Features:}

Movement in machines is controlled by the Push Buttons. The actuator comes into action when the controller presses the switch. Buttons are usually made up of either plastic or metals. The condition of the press catch may fit in with fingers or hands for basic use, or they may fundamentally be level. Press buttons usually have three sections that are actuator, stationary contacts, and scores.

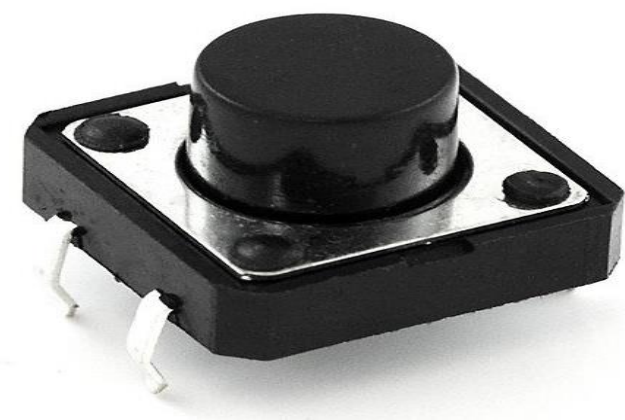

Fig. 28 Push Button

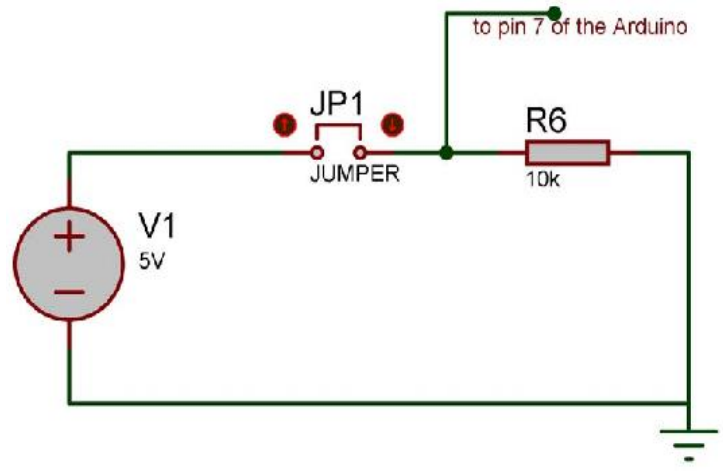

Fig. 29 Architecture Diagram of Push Button

\section{P. Integrated circuit (IC)}

\section{a) Definition:}

An IC is a little part, generally made of silicon that can hold numerous transistors, resistors, and capacitors. In view of the proposed application, the IC is delegated simple coordinated circuits, computerized incorporated circuits and blended incorporated circuits. It is a little chip that can work as an enhancer, oscillator, clock, microchip, or even PC memory.

\section{b) Features:}

The examples may contain numerous transistors, resistors and other electronic parts. It comprises of little squares of silicon, engraved with minuscule examples. The power our PCs, mobile phones, and music players. It makes everything from toaster stoves to autos progressively effective. As chip architects keep on improving the cutting edge,

it capacities into ever-littler bundles. The coordinated circuit (IC) is one of the most unpredictable things at any point made.

Published By:

Blue Eyes Intelligence Engineering DOI: 10.35940/ijeat.D8381.049420

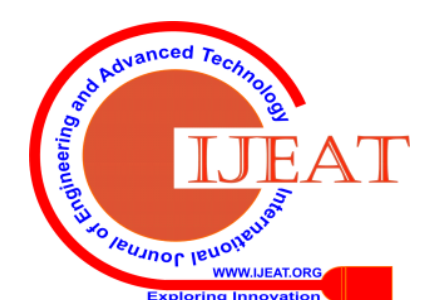




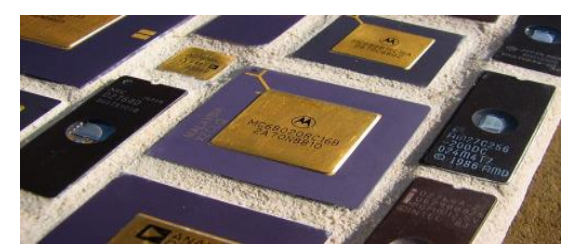

Fig. 30 IC

\section{Q. IC Sockets}

\section{a) Definition}

First IC connection is established for using IC chips. Because of welding, the heat produced may damage the IC chip. A device having a consolidated circuit uses a large amount of IC chips for connection.

\section{b) Features:}

PC motherboards utilize an attachment for the CPU, permitting you to pick your own processor for the board and overhaul the CPU. Programmable chips, for example, EPROMs or microcontrollers are put in IC attachments during prototyping, permitting the gadgets to be immediately expelled from the circuit for programming, and afterward reinserted for testing. Some incorporated circuits are amazingly delicate, and might be harmed by the warmth from binding, so to secure them they are put in IC attachments and furthermore for simple substitution if disappointments happen.

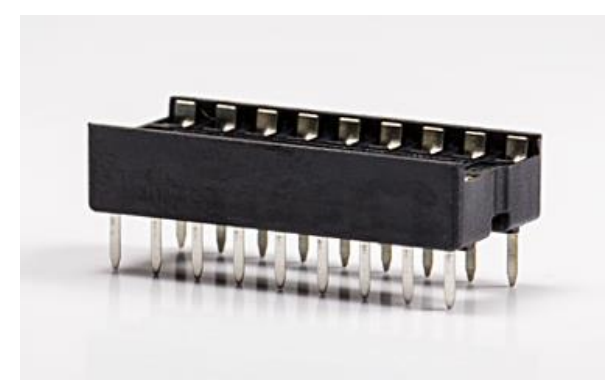

Fig. 31 IC Socket

\section{EXPERIMENTAL OUTPUT}

As explained in the implementation, when the transmitting module containing the accelerometer detects the tilt angle, a particular message is sent for the respective tilt angle. In this device, the message that is displayed when the accelerometer is turned right is "FOOD" and when the accelerometer turns left, it displays the message "WATER". When the accelerometer is turned upward, it displays the message "WASHROOM" and when the accelerometer is turned downward, it displays the message "HELP". Thus four different messages are delivered to the care-takers by the patient by tilting according to their needs.

\section{FUTURE SCOPE}

To enhance the salient features of this system, one of the features that can be added is the network part in which the messages can be sent via wi-fi or any sort of such network that increases the range for the transmitted signals. Another additional feature can be added in the domain of communication that is, making a phone call instead of messages. Sometimes, due to any sort of hardware failure, if the message has been failed to be sent, then a phone call may be initiated conveying the message that the patient is waiting for the respective nurse, doctor or the care-taker. This entire system may further be developed by introducing communication via any Android app which creates an interface for the patient-nurse communication.

\section{CONCLUSION}

This system is entirely automated without the need of any manual help from any sort of care-takers, may it be the nurse, doctor or the guardian itself. By introducing this system, the disabled and the paralytic patients are capable of being independent by performing small actions or movements for conveying their messages and requirements through this automated device which is capable of transmitting the messages in the form of signals to the corresponding caretaker. This makes it easy not only for the patient, but also for the care-takers, as they not necessarily need to be beside the patient all the time making the attenders also immobile indirectly. One of the main advantages of this system is its accuracy, as this system is quite accurate when it comes to

delivering the messages since there are only particular angles in which the messages can be sent. Thus, by the implementation of this entire system an easier form of inputs are obtained through a simple and easy-to-use device for the disabled and the paralyzed patients. This makes the communication process quite accurate, fast and effective. Hence a proficient approach has been implemented between the patient-nurse communications.

\section{REFERENCES}

1 Electronic Device Control Using Hand Gesture Recognition System For Differently Abled, Sanmukh Kaur, Anuranjana, Nivedita Nair, Amity School Of Engineering and Technology, Amity University, Uttar Pradesh, Noida, India.

2 Finger Movement based Attender Calling System for ICU Patient Management and Rehabilitation, Debeshi Dutta, Biswajeet Champaty, Kunal pal, Indranil Banerjee, Department of Biotechnology and Medical Engineering, NIT-Poutkela, Odisha, India.

3 SEEK: SIP-based Emergency Embedded frameworK supports elderly and disabled to perform emergency calls, Foteini Andriopoulou, Anastatios Fanariotis and Theofanis Orphanoudakis.

4 Integration of Tilt Sensors as a Device for Monitoring Rehabilitation Process, Safyzan Salim1,2,3, Wan Nurshazwani Wan Zakarial, Azrai Nizhan3 and M, Mahadi Abdul Jamil1 IDepartment of Electronic Engineering, Biomedical Modeling \& Simulation Research Group, Universiti Tun Hussein Onn Malaysia, Parit Raja 86400, Batu Pahat, Johor 2Communication Section Dept UniKL-BMI 3Kolej Kemahiran Tinggi MARA, Ledang, Johor

5 Multi-axis Stiffness Sensing Device for Medical Palpation,

6 Faragasso, A. Stilli, J. Bimbo, H.A. Wurdemann, K. Althoefer, Member, IEEE

7 Finger Motion Detection Glove Toward Human-Machine Interface, Ji-Hoon Suh*, Morteza Amjadi+, Inkyu Park+, and Hyung-Joun Yoo* *Department of Electrical Engineering, +Department of Mechanica Engineering, Mobile Sensor and IT Convergence Center (MOSAIC), Korea Advanced Institute of Science and Technology (KAIST), Daejeon, Republic of Korea

\section{AUTHORS PROFILE}

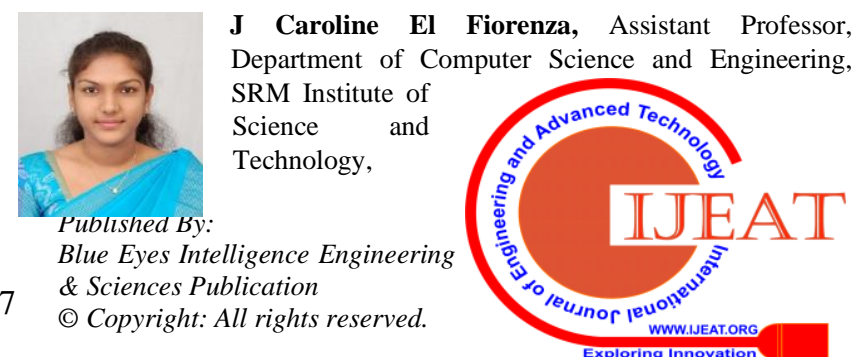


Chennai, India. Holds a PG degree M Tech (CSE) from SRM IST, Kattankulathur. Has more than six years' experience of teaching. She can be reached at email: caro.fiorenza@gmail.com

Kirthika Sivakumar, UG Scholar, B Tech, Department of Computer Science and Engineering, SRM Institute of Science and Technology, Chennai, India. She can be reached at email: kirthika_siv@yahoo.com.

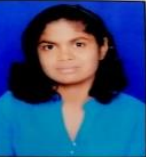

Barbie Das , UG Scholar, B Tech, Department of Computer Science and Engineering, SRM Institute of Science and Technology, Chennai, India. She can be reached at email:barbiedas204@gmail.com.

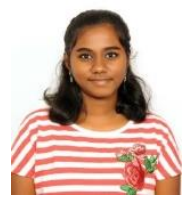

M Reene Stephanie, UG Scholar, B Tech, Department of Computer Science and Engineering, SRM Institute of Science and Technology, Chennai, India. She can be reached at email: reenestephanie@gmail.com.

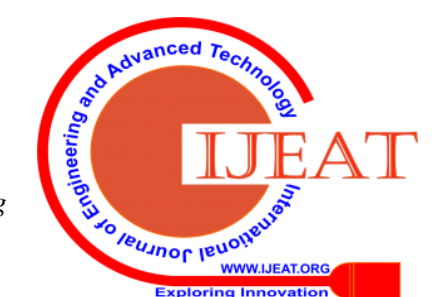

